\title{
Some Theorems and Results on Solution of Kind of Volterra Integral Equations
}

\section{Muayyad Mahmood Khalil}

Department of Mathematics, College of Education for Pure Sciences, Tikrit University, Tikrit, Iraq Email: medomath80@tu.edu.iq

(Received September 22, 2018; Accepted April 10, 2019; Available online June 01, 2020)

DOI: 10.33899/edusj.2020.165298, (C 2020, College of Education for Pure Science, University of Mosul.

This is an open access article under the CC BY 4.0 license (http://creativecommons.org/licenses/by/4.0/).

\section{Abstract}

We studied in this work a class of nonlinear Volterra integral equations with delay and present a sufficient condition for the bounded solution also we give an illustrative example which support the main result of this paper.

Keywords: Volterra, Time Delay, Integral Equation, Bounded Solution.

$$
\begin{aligned}
& \text { بعض المبرهنات والنتائج حول حل صنف من معادلات فولتيرا التكاملية } \\
& \text { مؤيد محمود خليل } \\
& \text { جامعة تكربت، كلية التربية للعلوم الصرفة، قسم الرياضيات }
\end{aligned}
$$

لقد درسنا في هذا البحث صنف معين من معادلات فولتيرا التكاملية غير الخطية من النوع المتباطئ وقدمنا الشرط الكافي ليكون الحل مقيداً واعطينا كذلك مثال توضيحي يدعم النتيجة الرئيسة للبحث. كلمات مفتاحية: فولتيرا، التأخير الزمني، معادلة تكاملية، حل مقيد.

إن معادلات فولتيرا التكاملية تضم أنواعاً كثيرة من الأنظمة الديناميكية وكمثال على ذلك المعادلات التفاضلية المتباطئة مع تباطؤ منته أو غير مقيد. لذلك فالعديد من النماذج التي ظهرت في الهندسة والتطبيقات العلمية الأخرى تكون مناسبة تماماً لهذه الفئة العامة من المعادلات وقد اهتممنا في بحثا هذا بالصفات النوعية الأساسية لمعادلات فولتيرا التكاملية المتباطئة غير الخطية ومن هذه الصفات تقييد الحلول ويعتبر تقييد الحلول سؤالً مهماً من كلا الناحيتين النظرية والتطبيقية بجانب 
حقيقة التقييد لذا فمن المهم ايضاً في تلك التطبيقات الحصول على قيد أعلى جيد للحل. لقد درس الباحثون والمؤلفون المعادلات التفاضلية والتكاملية المتباطئة أو ذات التأخير الزمني المتكرر كنماذج رياضية في العلوم الطبيعية والاقتصاد والهندس، وتم استخدام تلك النماذج لوصف انتشار وظواهر النقل وتقييد واستقرارية الحلول للمعادلات

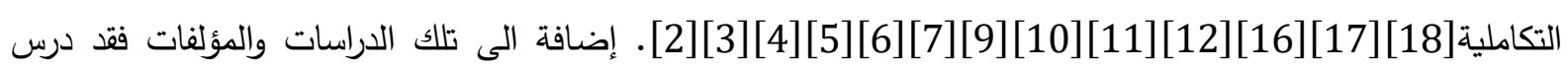
[1] Vecchio

$$
y(t)=g(t)+\int_{0}^{t} k(t, s) y(s) d s, t \in[0, T]
$$

حيث الخطية معتبراً أن أنويتها من النوع غير الملتف. ودرس Appleby وجماعته [8] التغير التام في انحلال الحل لمعادلة فولتيرا التكاملية من الصيغة

$$
R(t)=F(t)+\int_{0}^{t} H(t-s) R(s) d s
$$

حيث أعطى تقديرات لنسبة انحلال الحل في الفضاءات القابلة للتكامل. وفي [14] تمت دراسة تقييد الحلول للنظام

$$
x^{\prime}(t)=f(t, x(t)
$$

حيث $V:[0, \infty) \times R^{n} \rightarrow[0, \infty), a:[0, \infty) \rightarrow[0, \infty), b:[0, \infty) \rightarrow[0, \infty)$ وإن V تحقق شرط لبشتز محلياً في $x$ وأن

$$
a(|x|) \leq V(t, x) \leq b(|x|)
$$

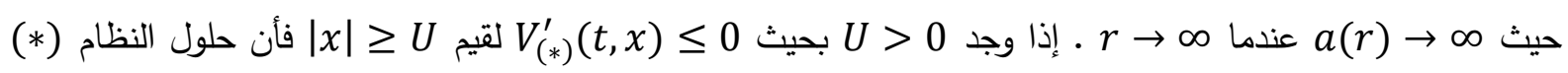

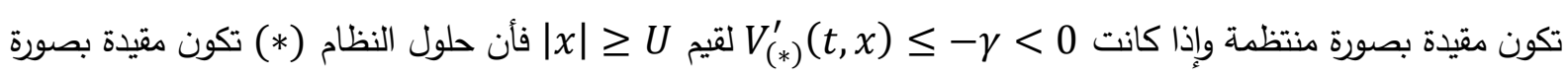
منتظمة لأقصى درجة. أما في [13] فقد تمت دراسة المعادلة التكاملية من الصيغة

$$
u^{p}(t)=L(t)+\int_{o}^{t} P(t-s) u(s) d s, t \geq 0, p \geq 1
$$

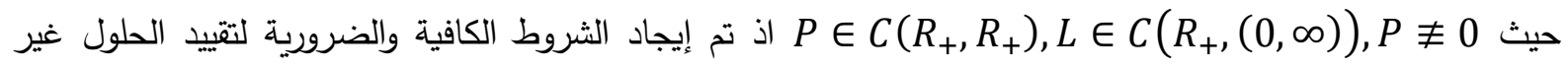
السالبة كما تم بيان شروط تقارب الحل بصورة محاذية لتحديد قيد الحل. وسنركز في دراستنا هذه على الحلول المقيدة لصنف من معادلات فولتيرا التكاملية غير الخطية من النوع المتباطئ حيث سنحاول استتاج الشروط الضرورية للحصول على الحلول المقيدة في الحالات التي لا يمكن أن تنطبق عليها القضية التي

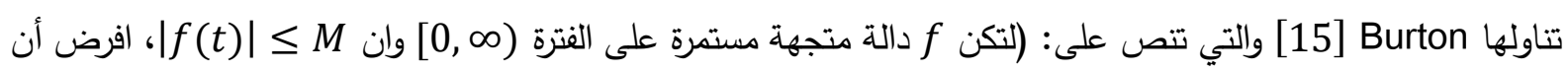
$\int_{0}^{t}|D(t, s)| d s \leq m$ s $D(t, s)$ لقيم

$$
x(t)=f(t)+\int_{0}^{t} D(t, s) x(s) d s
$$




\section{1. تعاريف أساسية}

لتكن لدينا معادلة فولتيرا التكاملية غير الخطية من النوع المتباطئ بالصيغة

$y(t)=\int_{o}^{t} f(t, s, y()) d s+.h(t)$

مع الشرط الابتدائي

$y(t)=\varphi(t), t \in\left[-t_{0}, 0\right]$

إذ 00 t 0 نقطة ثابتة وان الشروط الاتية متحققة:

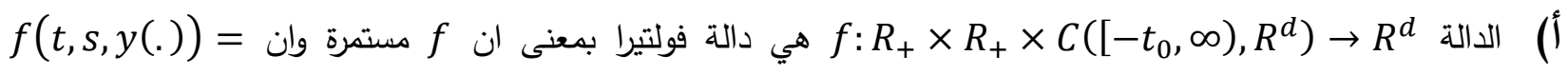

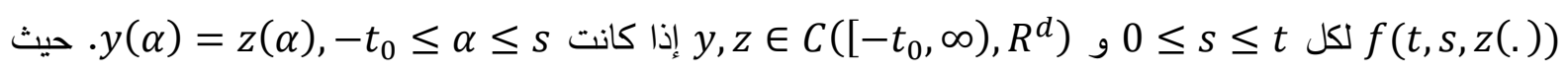

(real column هي مجموعة الاعداد الحقيقية غير السالبة و $R_{+}^{d}$ هو الفضاء من البعد d للمتجهات الحقيقية

.vectors)

بأل ألي

$\left|f_{i}(t, s, y()).\right| \leq k_{i}(t, s) \emptyset\left(\max _{-t_{0} \leq \vartheta \leq s}\|y(\vartheta)\|\right), i=1, \ldots, d$

متحقق عندما

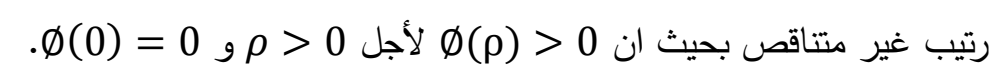

$$
\begin{array}{r}
\left.. h(t)=\left(h_{1}(t), \ldots, h_{d}(t)\right)^{T}, t \geq 0 \text { ث) التطبيق } 4: R_{+} \rightarrow R^{d} \text { مستمر }\right) \\
. \psi \in C\left(\left[-t_{0}, \infty\right), R^{d}\right)
\end{array}
$$

ان (.)

$f(t, s, y())=.g(t, s, y(s), 0 \leq s \leq t$

او ربما تحوي قيماً معتمدة على f من القيم التابعة للدالة (.)y • ويمكن وصف هذا بحد متأخر زمنياً ذو نقطة واحدة او على الثكل $f(t, s, y())=.g(t, s, y(s-\sigma(s)), 0 \leq s \leq t$

او بعدة نقاط متأخرة زمنياً

$f(t, s, y())=.g\left(t, s, y(s), y\left(s-\tau_{1}(s)\right), \ldots, y\left(s-\tau_{n}(s)\right)\right), 0 \leq s \leq t$

كذلك فإن التعبير ( $)$ 
$f(t, s, y())=.g\left(t, s, \int_{s-\tau}^{s} b(s, u) x(u) d u, 0 \leq s \leq t\right.$

أو ان اعتماد f على قيم سابقة من (.) بصورة اكثر عمومية. تعريف (2-2) لتكن الدوال Ø و i لأي

$\left|f_{i}(t, s, y()).\right| \leq k_{i}(t, s) \emptyset\left(\max _{-t_{0} \leq \vartheta \leq s}\|y(\vartheta)\|\right), i=1, \ldots, d$

متحقق عندما

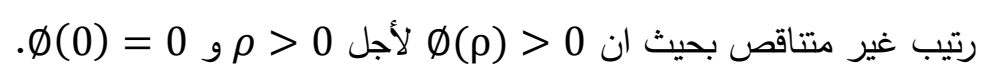

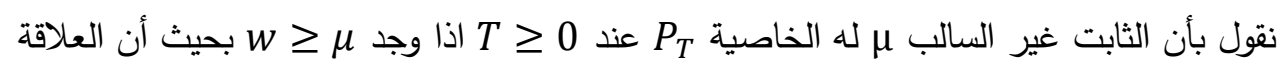

$\varnothing(\mu) \int_{0}^{T} k_{i}(t, s) d s+\emptyset(w) \int_{T}^{t} k_{i}(t, s) d s+\left|h_{i}(t)\right|<w, t \geq T, i=1, \ldots, d$

متحققة.

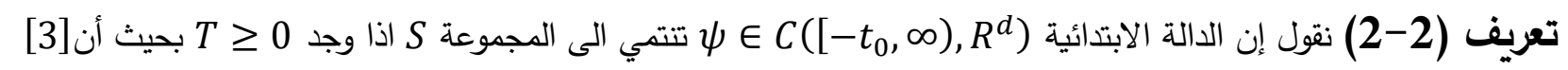
$\mu_{T}=\max _{-t_{0} \leq t \leq T}\|x\|$

له الخاصية $P_{T}$ عندما $\left.\rightarrow R^{d}\right)\left[-t_{0}, \infty\right.$ هي حل للمعادلتين (2.2) - (2.1). ملاحظة إذا وجد 0 م 0 وثابتين موجبين مثل $\mu_{T}, w$ بحيث ان (2.1) متحققة عندئذ:

$I_{T}=\max _{1 \leq i \leq d} \sup _{t \geq T} \int_{0}^{T} k_{i}(t, s) d s<\infty$

$J_{T}=\max _{1 \leq i \leq d} \sup _{t \geq T} \int_{T}^{t} k_{i}(t, s) d s<\infty$

$H_{T}=\max _{1 \leq i \leq d} \sup _{t \geq T}\left|h_{i}(t)\right|<\infty$

ان كلا الثرطين (2.5), (2.4) مكافئين للعلاقة 
$J_{0}=\max _{1 \leq i \leq d} \sup _{t \geq T} \int_{T}^{t} k_{i}(t, s) d s<\infty$

$$
\text { واذا كانت } 0=0
$$

$H_{0}=\max _{1 \leq i \leq d} \sup _{t \geq 0}\left|h_{i}(t)\right|<\infty$

\section{2. 2 النتيجة الرئيسة}

في هذا البند سنعطي الثروط اللازمة لوجود الحل المقيد للنظام (2.1)

مبرهنة (3-1) لتكن الشروط (أ)-(ث) متحققة ولتكن الدالة الابتدائية భ منتمية الى المجموعة S. عندئذ يكون الحل y لكلاً من (2.2) - منيداً.

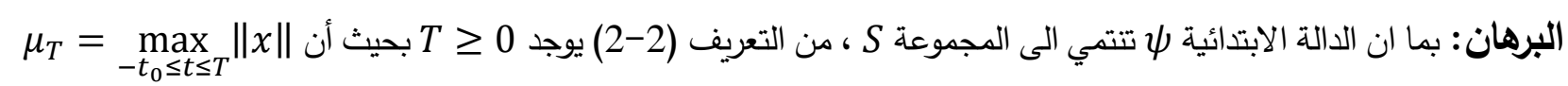
له الخاصية فمن المعادلة (2.1) نجد أن

$y(t)=\int_{o}^{T} f(t, s, y()) d s+.\int_{T}^{t} f(t, s, y() d s+.h(t), t \geq T$

بالتالي فالثرط (ب) وعندما d, d

$\left|y_{i}(t)\right| \leq \int_{o}^{T}\left|f_{i}(t, s, y()).\right| d s+\int_{T}^{t}\left|f_{i}(t, s, y()).\right| d s+\left|h_{i}(t)\right|$

$\leq \int_{o}^{T} k_{i}(t, s) \emptyset\left(\max _{-t_{0} \leq \vartheta \leq s}\|y(\vartheta)\|\right) d s+\int_{T}^{t} k_{i}(t, s) \emptyset\left(\max _{-t_{0} \leq \vartheta \leq s}\|y(\vartheta)\|\right) d s+\left|h_{i}(t)\right|$

$\leq \varnothing\left(\mu_{T}\right) \int_{o}^{T} k_{i}(t, s) d s++\int_{T}^{t} k_{i}(t, s) \emptyset\left(\max _{-t_{0} \leq \vartheta \leq s}\|y(\vartheta)\|\right) d s+\left|h_{i}(t)\right|, t \geq T$

ليكن $w \geq \mu_{T}$ إذ تتحقق العلاقة (2.3) عند $\mu=\mu_{T}$ عندئذ وبصورة خاصة فإن

$\varnothing\left(\mu_{T}\right) \int_{0}^{T} k_{i}(T, s) d s+\left|h_{i}(t)\right|<w, i=1, \ldots, d$ 


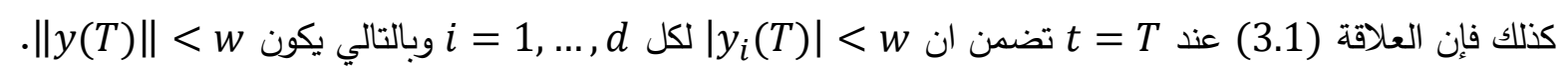

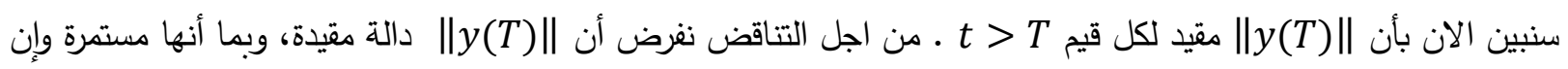

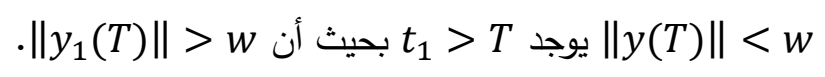
لتكن $\max _{-t_{0} \leq \alpha \leq \hat{t}}\|y(\alpha)\|=\|y(\hat{t})\|=w$

لهذا السبب يوجد $i$ إذ أن للخاصية P Pحصل على

$w=\left|y_{i}(\hat{t})\right| \leq \varnothing\left(\mu_{T}\right) \int_{o}^{T} k_{i}(\hat{t}, s) d s+\int_{T}^{\hat{t}} k_{i}(\hat{t}, s) \emptyset\left(\max _{-t_{0} \leq \alpha \leq s}\|y(\alpha)\|\right) d s+\left|h_{i}(\hat{t})\right|$

$\leq \emptyset\left(\mu_{T}\right) \int_{o}^{T} k_{i}(\hat{t}, s) d s+\emptyset(w) \int_{T}^{\hat{t}} k_{i}(\hat{t}, s) d s+\left|h_{i}(\hat{t})\right|$

$<w$

وهذا تناقض. لذا فإن الحل للمعادلة (2.1) يكون مقيداً بـ w.

3. 3عض الحالات الخاصة بالمبرهنة (1-3)

في هذا البند سنعطي بعض الحالات التي تخص المبرهنة الرئيسة في بحثا هذا وذلك من خلال فرض ان مكونات الدالة غير الخطية في المعادلة (2.1) يمكن تقديرها بدالة مثل فئلt هناك ثلاث حالات لقيمة p وهي:

$$
\begin{array}{r}
.0<p<1(\mathbf{1} \\
. p=1(\mathbf{2} \\
. p>1(\mathbf{3}
\end{array}
$$

المبرهنات التالية توضح الثروط الكافية ليكون الحل مقيدا لكل قيمة من قيم p أعلاه

مبرهنة (4-1) لتكن الثروط من (أ) الى (ث) متحققة و ان (2.7) (2.8) (2.1) متحققة فإن كل حلول المعادلة (ل) منيدة. 


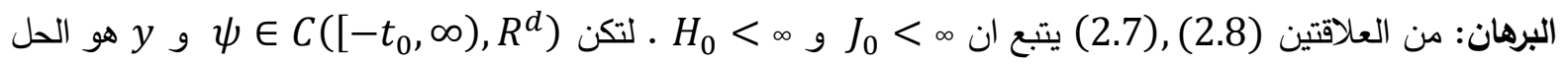
المطابق لـ (2.2), (2.1) . لأي ||

${ }^{p-1} J_{0}+\frac{1}{w} H_{0}<1$

$w^{p} J_{0}+H_{0}<w$

من تعريف

$w^{p} \int_{0}^{t} k_{i}(t, s) d s+\left|h_{i}(t)\right| \leq w^{p} J_{0}+H_{0}<w, t \geq 0, i=1, \ldots, d$

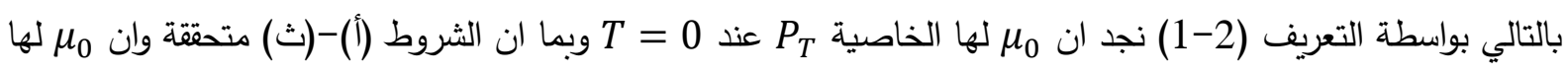
الخاصية P Pندئذ وبوساطة المبرهنة (3-1) يكون الحل مقيد. انتهى البرهان

لنتوصل الى المبرهنة الثانية نفرض ان لدينا معادلة فولتيرا من الصيغة:

$y(t)=\int_{o}^{t} k(t, s) y^{p}(s-(\tau(s)) d s+h(t), t \geq 0$

مع الشرط

$y(t)=\psi, t \in\left[-t_{0}, 0\right]$

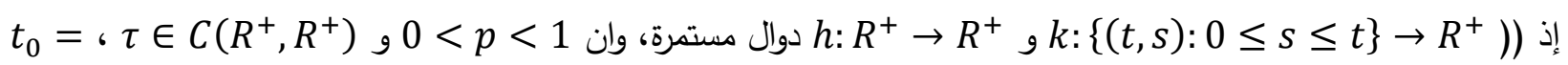

(ج) $\left(\left(\psi \in C\left(\left[-t_{0}, 0\right],[0, \infty]\right)\right.\right.$ ونته $-\inf _{t \geq 0}\{t-\tau(t)\}>0$

ان الثرط أعلاه (ج) يضمن بأن تكون الحلول لكل من (4.3), (4.2) موجبة.

نتيجة المبرهنة التالية تبين الثرط الضروري والكافي لتكون الحلول الموجبة للمعادلة (4.2) مقيدة. الجزء الضروري من المبرهنة القادمة مشابه للنتيجة التي توصل اليها Lipovan [13] والتي تم برهانها من اجل معادلة تكاملية من النوع الملتق. مبرهنة (4-2) افرض أنه لدينا الثرط (ج) وان

$\lim _{t \rightarrow \infty} \inf \left(\int_{o}^{t} k(t, s) d s+h(t)\right)>0$ 
$\int_{o}^{t} k(t, s) d s+h(t)>0, t \geq 0$

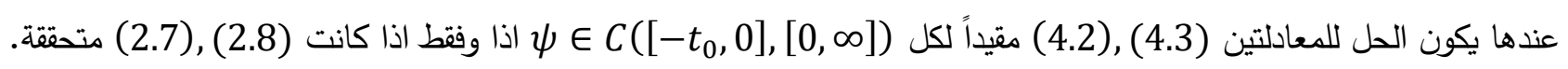
البرهان: افرض ان (2.8), (2.7) متحققة ، من الواضح أنه ومن مبرهنة (4-1) فالحل لكل من (4.2) مقيد لكل قيم .$\psi \in C\left(\left[-t_{0}, 0\right],[0, \infty]\right)$ بصورة عكسية ، ليكن الحل y مقيداً على +R. في البداية سنثبت ان 0 >

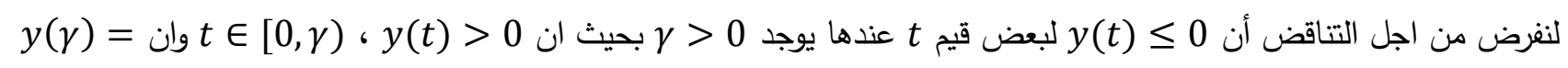
0

$\int_{0}^{\gamma-\varepsilon} k(\gamma, s) d s+h(\gamma)>0$

من (4.2) عند

$0=y(\gamma)$

$=\int_{0}^{\gamma} k(\gamma, s) y^{p}(s-\tau(s)) d s+h(\gamma)$

$\geq \int_{0}^{\gamma-\varepsilon} k(\gamma, s) y^{p}(s-\tau(s)) d s+h(\gamma)$

$\min _{-t_{0} \leq \mu \leq \gamma-\varepsilon} y^{p}(\mu) \int_{0}^{\gamma-\varepsilon} k(\gamma, s) d s+h(\gamma)$

$\geq\left(\int_{0}^{\gamma-\varepsilon} k(\gamma, s) d s+h(\gamma)\right)\left(\min _{-t_{0} \leq \mu \leq \gamma-\varepsilon} y^{p}(\mu), 1\right)$

$>0$

وهذا تتاقض.

بصورة واضحة فإن قيمة y الموجبة تقودنا الى 


$$
y(t) \geq h(t), \forall t \geq 0
$$

بالتالي فالثرط (2.8) متحقق.

الان سنثبت (2.7) ، لأي 0 إن

$y(t) \geq \int_{0}^{T^{*}} k(t, s) y^{p}(s-\tau(s)) d s \geq \min _{-t_{0} \leq \mu \leq T^{*}} y^{p}(\mu) \int_{0}^{T^{*}} k(t, s) d s+h(\gamma)$

لذا فإن

$\sup _{t \geq 0} \int_{0}^{T^{*}} k(t, s) d s<\infty$

نعرف الان

$m=\lim _{t \rightarrow \infty} \inf y(t)$

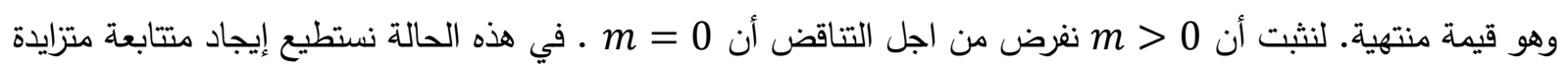
بحيث أن $\left(t_{n}\right)_{n \geq 1}$

$y\left(t_{n}\right)=\inf _{-t_{0} \leq t \leq t_{n}} y(t)>0$

وإن من (4.2) عند قيمة

$$
\begin{aligned}
& y\left(t_{n}\right)=\int_{0}^{t_{n}} k\left(t_{n}, s\right) y^{p}(s-\tau(s)) d s+h\left(t_{n}\right) \\
& \geq \int_{0}^{t_{n}} k\left(t_{n}, s\right) \inf _{-t_{0} \leq \mu \leq t_{n}} y^{p}(\mu) d s+h\left(t_{n}\right) \\
& =y^{p}\left(t_{n}\right) \int_{0}^{t_{n}} k\left(t_{n}, s\right) d s+h\left(t_{n}\right)
\end{aligned}
$$


$y^{1-p}\left(t_{n}\right) \geq \int_{0}^{t_{n}} k\left(t_{n}, s\right) d s+\frac{h\left(t_{n}\right)}{y^{p}\left(t_{n}\right)}$

ومن اجل قيمة كبيرة كفاية لـ n إذ أن 1 > 1 إن 0 ينتج لدينا

$y^{1-p}\left(t_{n}\right) \geq \int_{0}^{t_{n}} k\left(t_{n}, s\right) d s+h\left(t_{n}\right)$

بأخذ الغاية للمتباينة أعلاه

$\lim _{n \rightarrow \infty} \int_{0}^{t_{n}} k\left(t_{n}, s\right) d s+h\left(t_{n}\right)=0$

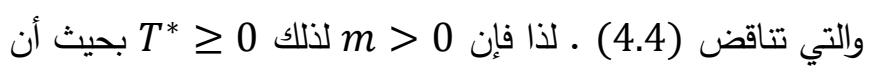

$y(t) \geq \frac{1}{2} m>0, t \geq T^{*}$

بالنتيجة

$y(t)=\int_{o}^{t} k(t, s) y^{p}(s-\tau(s)) d s+h(t)$

$\geq \int_{T^{*}}^{t} k(t, s) y^{p}(s-\tau(s)) d s$

$\geq \frac{1}{2^{p}} m^{p} \int_{T^{*}}^{t} k(t, s) d s, t \geq T^{*}$

بالاستفادة من كون الحل y مقيد نحصل على

$\sup _{t \geq T^{*}} \int_{T^{*}}^{t} k(t, s) d s<\infty$

والمتباينة أعلاه والمعادلة (4.6) تضمن تحقيق الشرط (2.7).

مبرهنة (4-3) افرض أن الثروط (أ)-(ب) متحققة وان 0 (t) =t, t 0 عندئذ جميع الحلول للمعادلة (2.1) مقيدة وإذا كان

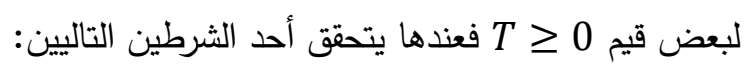


(1) (2.6) (2.4) متحققة وأن من (1) (2.4)

$J_{T}=\max _{1 \leq i \leq d} \sup _{t \geq T} \int_{T}^{t} k_{i}(t, s) d s<1, i=1, \ldots, d$.

(2)

$J_{T}=\max _{1 \leq i \leq d} \sup _{t \geq T} \int_{T}^{t} k_{i}(t, s) d s=1, \quad \int_{T}^{t} k_{i}(t, s) d s<1$

$\sup _{t \geq T}\left(1-\int_{T}^{t} k_{i}(t, s) d s\right)^{-1} \int_{0}^{T} k_{i}(t, s) d s<\infty$

$\sup _{t \geq T}\left(1-\int_{T}^{t} k_{i}(t, s) d s\right)^{-1}\left|h_{i}(t)\right|<\infty$

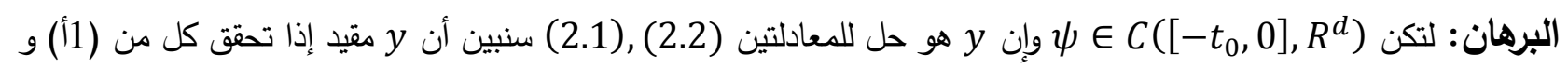
(2) (ب). ليكن

$$
\mu_{T}=\max _{-t_{0} \leq t \leq T}\|y(t)\|
$$

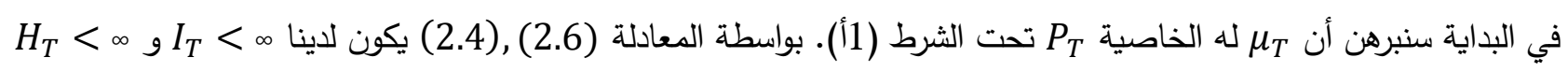
لهذا يوجد

$\left(1-J_{T}\right)^{-1}\left(\mu_{T} I_{T}+H_{T}\right)<w$

وكذلك

$\mu_{T} I_{T}+H_{T}<\left(1-J_{T}\right) w$

بالتالي ولكل قيم d, d

$\mu_{T} \int_{0}^{T} k_{i}(t, s) d s+\left|h_{i}(t)\right| \leq \mu_{T} I_{T}+H_{T}<\left(1-J_{T}\right) w \leq\left(1-\int_{T}^{t} k_{i}(t, s) d s\right) \mathrm{w}$ 
$\mu_{T} \int_{0}^{T} k_{i}(t, s) d s+w \int_{T}^{t} k_{i}(t, s) d s+\left|h_{i}(t)\right|<w, t \geq T, i=1, \ldots, d$

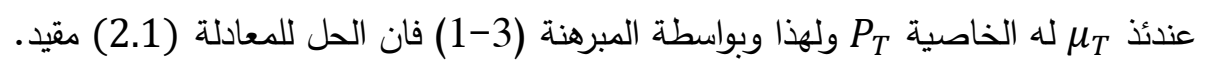
سنثبت لاحقا ان $\mu_{T}$ له الخاصية $P_{T}$ اذا كان الشرط (2ب) متحقق. لكل قيم $t \geq T$

$\left(1-\int_{T}^{t} k_{i}(t, s) d s\right)^{-1}>0, i=1, \ldots, d$

فإن (4.9) ) تضمن لنا بأن

$\sup _{t \geq T}\left(1-\int_{T}^{t} k_{i}(t, s) d s\right)^{-1}\left\{\mu_{T} \int_{0}^{T} k_{i}(t, s) d s+\left|h_{i}(t)\right|\right\}<\infty$

عندئذ يوجد $w \geq \mu_{T}$

$\left(1-\int_{T}^{t} k_{i}(t, s) d s\right)^{-1}\left\{\mu_{T} \int_{0}^{T} k_{i}(t, s) d s+\left|h_{i}(t)\right|\right\}<w, t \geq T$

والذي يؤدي الى

$\mu_{T} \int_{0}^{T} k_{i}(t, s) d s+\mathrm{w} \int_{T}^{t} k_{i}(t, s) d s+\left|h_{i}(t)\right|<w, t \geq T, i=1, \ldots, d$

عند ذلك فإن $\mu_{T}$ له الخاصية $P_{T}$ وبالتالي وحسب المبرهنة (3-1) فإن الحل y للمعادلة (2.1) يكون مقيداً.

مبرهنة (4-4) افرض ان الثروط (أ)-(ب) متحققة وان 0 (t) 0 (t) 0 ولتكن |l (2.1) مقيد اذا تحققت احدى الشروط التالية:

$$
\begin{gathered}
. H_{0}<\frac{p-1}{p}\left(\frac{1}{p^{J_{0}}}\right)^{\frac{1}{p-1}},\|\psi\|_{t_{0}}=\left(\frac{1}{p^{J_{0}}}\right)^{\frac{1}{p-1}}(1) \\
. H_{0}<\|\psi\|_{t_{0}}-J_{0}\left(\|\psi\|_{t_{0}}\right)^{p}(2) \\
\text { البرهان: افرض ان (2.8), (2.7) متحققة })
\end{gathered}
$$




$$
\begin{aligned}
& \text { فأن } H_{0}<\frac{p-1}{p}\left(\frac{1}{p^{J_{0}}}\right)^{\frac{1}{p-1}}=\left(\frac{1}{p^{J_{0}}}\right)^{\frac{1}{p-1}}-J_{0}\left(\frac{1}{p^{J_{0}}}\right)^{\frac{p}{p-1}},\|\psi\|_{t_{0}}=\left(\frac{1}{p^{J_{0}}}\right)^{\frac{1}{p-1}} \text { (1) } \\
& w=\left(\frac{1}{p^{J}}\right)^{\frac{1}{p-1}}
\end{aligned}
$$

$H_{0}<w-J_{0} w^{p}$

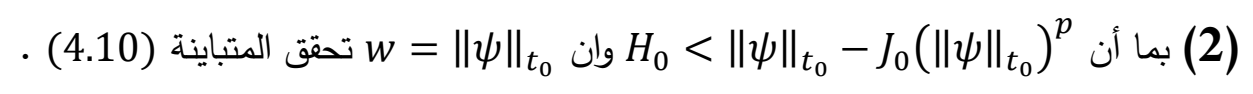
بالتالي ففي كلتا الحالتين فان الشرط (2.3) يتحقق عند 0 = $T$ لذلك (3-1-1) متحققة وعليه فإن الحل للمعادلة (2.1) مقيد.

\section{4. مثال تطبيقي}

$$
\text { لنوضح المبرهنة (4-3) في حالة متى ما كان الشرط (لكل قيم }
$$

$J_{T}=\max _{1 \leq i \leq d} \sup _{t \geq T} \int_{T}^{t} k_{i}(t, s) d s=1, \quad \int_{T}^{t} k_{i}(t, s) d s<1$

$\sup _{t \geq T}\left(1-\int_{T}^{t} k_{i}(t, s) d s\right)^{-1} \int_{0}^{T} k_{i}(t, s) d s<\infty$

$\sup _{t \geq T}\left(1-\int_{T}^{t} k_{i}(t, s) d s\right)^{-1}\left|h_{i}(t)\right|<\infty$

متحققاً لقيمة كبيرة كفاية لـ T.

لتكن لدينا المعادلة التالية

$y(t)=\int_{0}^{t} \frac{2}{1-\frac{1}{2} e^{-2|t-\ln 2|}} e^{-2(t-s)} y(s) d s+c e^{-2 t}, t \geq 0$

$$
k(t, s)=\frac{2}{1-\frac{1}{2} e^{-2|t-\ln 2|}}, t_{0}=0 \text { لاينا هنا } c \in R, h(t)=c e^{-2 t}
$$

من الواضح أن

$\sup _{t \geq 0}|h(t)|=|c|<\infty$ 


$$
\begin{aligned}
& \int_{0}^{t} k(t, s) d s=\int_{0}^{t} \frac{2}{1-\frac{1}{2} e^{-2|t-\ln 2|}} e^{-2(t-s)} d s \\
& =\frac{1}{1-\frac{1}{2} e^{-2|t-\ln 2|}}\left(1-e^{-2 t}\right)
\end{aligned}
$$

$\lim _{t \rightarrow \infty} \int_{0}^{t} k(t, s) d s=1$

لنضع 2 فان

$\int_{0}^{T_{1}} k\left(T_{1}, s\right) d s=\frac{1}{\left(1-\frac{1}{2}\right)}\left(1-e^{-2 \ln 2}\right)=\frac{3}{2}>1$

وهذا يعني ان

$\sup _{t \geq 0} \int_{0}^{t} k(t, s) d s=M>1$

لذا فإن شروط المبرهنة (4-3) لا تتحقق عندما 0 = إذا أخذنا

$\int_{T}^{t} k(t, s) d s=\frac{1-25 e^{-2 t}}{1-2 e^{-2 t}}<1, t \geq T$

$$
\sup _{t \geq 0} \int_{T}^{t} k(t, s) d s=1
$$


$\left(1-\int_{T}^{t} k(t, s) d s\right)^{-1} \int_{0}^{T} k(t, s) d s=\frac{24 e^{-2 t}\left(1-2 e^{-2 t}\right)}{23 e^{-2 t}\left(1-2 e^{-2 t}\right)}=\frac{24}{23}, t \geq T$

$\left(1-\int_{T}^{t} k(t, s) d s\right)^{-1}|h(t)|=\frac{\left(1-2 e^{-2 t}\right)}{23 e^{-2 t}}|c| e^{-2 t}, t \geq T$

لذا فإن الشرط الثاني للمبرهنة (4-3) يتحقق بهذه القيمة لـ T ، لذلك فالحل y مقيد.

نلاحظ هنا أن القضية (1.4.2) في [15] لا يمكن تطبيقها لهذا المثال لكن الثروط الكافية التي حصلنا عليها خلال البحث يمكن استعمالها.

\section{5. الاستنتاجات}

إن النتيجة الرئيسة لهذا البحث (مبرهنة (3-1)) قد أعطت الشروط الكافية لضمان تقييد الحلول لفئة كبيرة من معادلات فولتيرا التكاملية ذات التأخير الزمني والتي اعتمدت على متباينة خاصة وتقنية المقارنة كما أعطت المبرهنة (4-1) ضمان وجود الحل المقيد لمعادلات فولتيرا التكاملية في حالة كونها شبه خطية وبينت المبرهنة (4-2) الثروط الضرورية والكافية للحلول المقيدة في حالة المعادلات شبه الخطية والملتفة عددياً (scalar convolution) والتي توضح ان الثروط المعطاة ليست كافية وحسب وانما ضرورية كذلك ليكون الحل مقيداً بالإضافة الى ما سبق فقد تم إيضاح تقييد الحلول لمعادلات فولتيرا التكاملية الخطية والتي يمكن تطبيقها في الحالة الحرجة من خلال المبرهنة (4-3) واخيراً وليس اخراً بينت المبرهنة (4-4) الثروط الكافية للحلول المقيدة لمعادلات فولتيرا التكاملية فوق الخطية وفي النهاية تم إعطاء مثال توضيحي اثبتنا من خلاله انه يمكن تطبيق النتائج التي توصلنا اليها في حالة ان الطريقة المعروفة التي اعتمدها Burton [15] لا يمكن تطبيقها. 


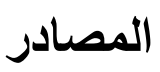

[1] A. Vecchio, J. Int. Eqs. Appl. 12 no. 4, 449-465 (2000),

[2] C. Corduneanu, "Integral Equations and Stability of Feedback Systems", 1st Ed. Academic Press, New York and London (1973).

[3] C. Corduneanu, "Integral Equations and Applications", Reissue edition. Cambridge University Press (2008).

[4] C. Cuevas and M. Pinto, Journal of Computational and Applied Mathematics, Volume 113, Issues 1-2, Pages 217-225, 1 January (2000).

[5] E.N. Chukwu, "Stability and Time-Optimal Control of Hereditary Systems", Volume 188,1st Ed. Academic Press (1992).

[6] H. Brunner, Collocation Methods for Volterra Integral and Related Functional Differential Equations, 1st Ed. Cambridge University Press, New York (2004).

[7] J.A.D. Appleby, I. Gyori, and D. Reynolds, Journal of Difference Equations and Applications Volume 12, 1257-1275 (2006).

[8] J.A.D. Appleby, István Gyori, David W. Reynolds, J. Math. Anal. Appl. 320 56-77 (2006).

[9] J. Chiasson and J.J. Loiseau, "Applications of Time Delay Systems", 1st Ed. Springer, Berlin, Heidelberg (2007).

[10] K. Gopalsamy, "Stability and Oscillation in Delay Differential Equations of Population Dynamics", 1st Ed. Springer (1992).

[11] M. Rahman, "Integral Equations and their Applications", 1st Ed. WIT Press, USA, Canada and Mexico (2007).

[12] N. MacDonald, "Time-lags in Biological Models, Lecture notes in Biomathematics", 1st Ed. Springer, Berlin (1978).

[13] O. Lipovan, On the asymptotic behavior of solutions to some nonlinear integral equations of convolution type. Dynamics of Continuous, Discrete \& Impulsive Systems. Series A: Mathematical Analysis, 16 (2), 147-154 (2009). 
[14] T.A. Burton, Boundedness in functional differential equations, Funkcialaj Ekvacioj 25, 51-77 (1982).

[15] T.A. Burton, "Volterra Integral and Differential Equations", 2nd Ed. Elsevier (2005).

[16] T. Erneux, "Applied Delay Differential Equations", 1st Ed. Springer (2009).

[17] T. Insperger and G. Stepan, "Semi-discretization for Time-delay Systems", Stability and Engineering Applications, 1st Ed. Springer (2011).

[18] Y. Kuang, "Delay Differential Equations with Applications in Population Dynamics", 1st Ed. Academic Press, Boston (1993). 